\title{
A New Polymeric Peroxycarbamate: Synthesis, Decomposition and Its Use of Polymerization Initiator for Methylstyrene
}

\author{
Huseyin Yildirim, Ahmet YiLmazturk, Bahattin M. BAYSAL, \\ and Yusuf YAGCI* \\ University of Yildiz, Department of Chemistry, Sisli, \\ Istanbul 80750, Turkey \\ * Technical University of Istanbul, Department of Chemistry, \\ Maslak, Istanbul 80626, Turkey
}

(Received August 8, 1988)

\begin{abstract}
A new polymeric peroxycarbamate (PPC), having a substituted cycloaliphatic moiety, was synthesized using the corresponding diisocyanate and a dihydroperoxide. The thermal decomposition kinetics of this PPC were studied in 1,4-dioxane solution at 80,90 , and $100^{\circ} \mathrm{C}$. The decomposition reaction was found to proceed according to first order kinetics with activation energy of $125 \mathrm{~kJ} \mathrm{~mol}^{-1}$. Furthermore, this PPC was used to initiate the bulk polymerization of methylstyrene at $80^{\circ} \mathrm{C}$ and shown to be an effective initiator with negligible chain transfer to the initiator.

KEY WORDS Polymeric Peroxycarbamate / Block Copolymer /

Chain Transfer
\end{abstract}

Peroxycarbamates (PC) are synthesized by utilising well established techniques of polyurethane chemistry. The polymeric and dimeric PCs were used as free radical initiators in the polymerization and block copolymerization of vinyl monomers. ${ }^{1-3}$ Block copolymerization is a two-step procedure and involves the sequential decomposition of peroxy linkages introduced in the initiator. We have recently extended ${ }^{4}$ this procedure to cation susceptible monomers such as butyl vinyl ether. In these processes, macroradicals, obtained by the thermolysis of polymeric peroxycarbamates (PPC) or polymers having residual peroxy bonds, are converted to carbocations by the use of organic salts. Although PPCs are efficient multipurpose initiators, chain transfer reactions to the initiator have deterred to the use of these initiators. ${ }^{5-7}$ Obviously, these reactions lead to the formation of polymers having no peroxy bonds in the main chain and homopolymer formation would be unavoid- able when used for the synthesis of block copolymers.

In this paper, we describe the synthesis and decomposition kinetics of a new PPC as well as the initiation of methylstyrene polymerization by this initiator, which revealed that transfer to the initiator was negligible.

\section{EXPERIMENTAL}

\section{Materials}

3-Isocyanatomethyl-3,5,5-trimethyl cyclohexyl isocyanate (IPDI) was a product of Fluka A.G. Its purity, determined by isocyanate analysis, was more than $95 \%$.

2,5-Dimethyl-2,5-dihydroperoxyhexane, (Luperox 2,5-2,5) was supplied by Lucidol Division, Penwalt Corp. It was recrystallised from carbon tetrachloride. The peroxygen content was $98 \%$ of the theoretical value.

Dibutyltin dilaurate (Carstan DBTDL, T12) was a product of Fluka A.G. and was used 
as received.

Methylstyrene monomer contained $60 \%$ of the meta-isomer and $40 \%$ of the para-isomer. This monomer was succesively washed with $10 \%$ aqueous $\mathrm{NaOH}$ solution and distilled water several times. After drying over calcium chloride, it was fractionally distilled under vacuum.

All solvents were purified according to conventional drying and distillation procedures. ${ }^{8}$

\section{Analyses}

Isocyanate analyses were performed by reacting a known amount of material with standard di- $n$-butylamine solution in toluene and back-titrating the excess amine with standard $\mathrm{HCl} .{ }^{9}$ The peroxy contents were determined by iodometric methods. ${ }^{10}$ IR spectra were recorded on a Perkin Elmer, Model 177 IR spectrometer. GPC chromatograms were obtained using A Knauer M64 instrument and using methylethylketone as the eluent and the flow rate was $1 \mathrm{ml} \mathrm{min}^{-1}$. Molecular weights were calculated according to polystyrene standard samples.

\section{PROCEDURES}

\section{Preparation of $P P C$}

$15 \mathrm{mmol}$ diisocyanate (IPDI) and $15 \mathrm{mmol}$ dihydroperoxide (Luperox 2,5-2,5) were reacted in $60 \mathrm{ml}$. dry methylene chloride at room temperature under dry nitrogen atmosphere in the dark for $72 \mathrm{~h}$. The chain extension reaction was carried out in the presence of T- 12 catalyst $(0.05 \mathrm{~g}) .15 \mathrm{mmol}$ IPDI was then added to this solution in order to cover both ends of the PC with isocyanate end groups. The reaction was conducted at room temperature for $48 \mathrm{~h}$. The isocyanate end groups were capped with $15 \mathrm{ml}$ isopropanol by reacting for $12 \mathrm{~h}$ at room temperature. The product was precipitated by petroleum ether $(400 \mathrm{ml})$, filtered, washed and dried in a vacuum oven at room temperature. Yield, $68.7 \%$; peroxygen content, $11.7 \% ; \bar{M}_{w}$ (from the peroxygen content), $1263.5 \mathrm{~g} \mathrm{~mol}^{-1}$; $\bar{M}_{n}$ (by a cryoscopic method), $1170.8 \mathrm{~g} \mathrm{~mol}^{-1}$. The chemical structure of PPC can be represented as follows.

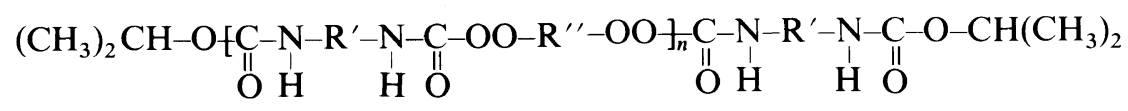<smiles>[R]CC1(C)CC[C]C(C)(C)C1</smiles>

\section{Thermal Decomposition Studies}

The thermal decomposition kinetics of PPC were studied in 1,4-dioxane at 80, 90, and $100^{\circ} \mathrm{C}$. Weighed amounts of samples were dissolved in the solvent and $10 \mathrm{ml}$ of these solutions were degassed on a high vacuum line by the freeze-thaw method, sealed and put into a constant temperature bath for the desired time intervals. After the reaction, the tubes were broken, the contents poured into benzene and the amount of unreacted peroxygen determined by iodometric titration. 


\section{Polymerization Procedures}

Given amounts of PPC and methylstyrene were introduced into pyrex reaction tubes and evacuated in the usual manner. The tubes were then sealed and put into a constant temperature bath at $80^{\circ} \mathrm{C}$. At the end of the reaction the mixtures were dissolved in toluene and precipitated in methanol several times. The polymer samples were dried in a vacuum oven. The conversions were deliberately kept below $10 \%$ so as to obtain reliable kinetic analysis of the data. The rate, $R_{\mathrm{p}}$, and avarage degree of polymerization, $\bar{P}_{n}$, were calculated from conversion-time data and dilute solution viscosity measurements, respectively. The viscometric equation used was:

$$
[\eta]=7.36 \times 10^{-5} \bar{M}_{n}^{0.76} \mathrm{dlg}^{-1}
$$

given for benzene solutions at $30^{\circ} \mathrm{C}$ for poly(methylstyrene) samples. ${ }^{11}$

\section{RESULTS AND DISCUSSION}

The synthesis of PPC was achieved by using a general procedure of polyurethane chemistry. The chain extension reaction was conducted at room temperature in order to prevent the thermal cleavage of the peroxygen bonds. At this step, dibutyltin dilaurate (T-12) was added as catalyst causing a significant increase in the reaction rate. Special care was taken for the removal of unreacted material from the product. IR spectroscopy was routinely used to follow the reactions by monitoring the disappearence of the ( $\mathrm{NCO}$ ) and $(\mathrm{OOH})$ bands at around 2250 and $3520 \mathrm{~cm}^{-1}$ and the appearence of $(\mathrm{NH})$ and $(\mathrm{C}=\mathrm{O}) \mathrm{ab}$ sorptions at around 3440 and $1730 \mathrm{~cm}^{-1}$, respectively. Functional group analysis also confirmed the formation of the predicted structure since the agreement between experimental and theoretical results was fairly good.

The thermal decomposition kinetics of PPC $\left(10^{-2} \mathrm{~mol}^{-1}\right)$ were studied by determining the amount of unreacted peroxygen groups as a function of time. First-order plots of $\ln \left(C_{0} / C\right)$

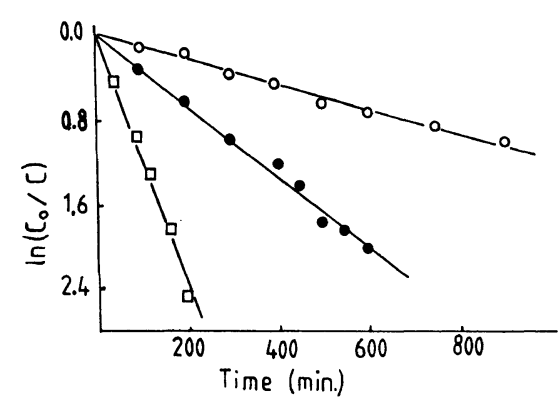

Figure 1. First-order plot for thermal decomposition of polymeric peroxycarbamate: $(\mathrm{O}), 80^{\circ} \mathrm{C}$; (O), $90^{\circ} \mathrm{C}$; $(\square), 100^{\circ} \mathrm{C}$.

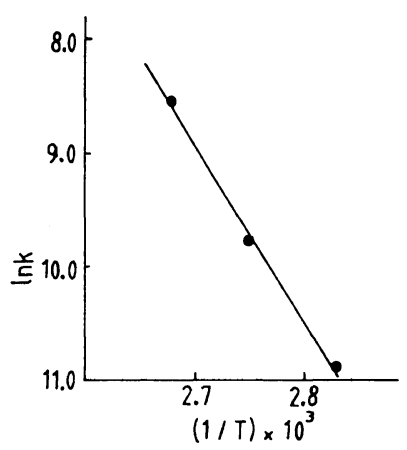

Figure 2. Arrhenius plot for decomposition rate constant $\left(k_{\mathrm{d}}\right)$ of polymeric peroxycarbamate.

versus $t$ for PPC are illustrated in Figure 1, where $C_{0}$ and $C$ denote the initial concentration and concentration at time $t$ of the PC groups. The decomposition rate constants $\left(k_{\mathrm{d}}\right)$ at 80,90 , and $100^{\circ} \mathrm{C}$ were calculated from the slopes of those straight lines. The values are $k_{\mathrm{d}}=1.88 \times 10^{-5}\left(80^{\circ} \mathrm{C}\right), 5.67 \times 10^{-5}\left(90^{\circ} \mathrm{C}\right)$, and $19.91 \times 10^{-5} \mathrm{~s}^{-1}\left(100^{\circ} \mathrm{C}\right)$. The activation energy $\left(E_{\mathrm{a}}\right)$ and frequency factor $(A)$ values were estimated to be $125 \mathrm{~kJ} \mathrm{~mol}^{-1}$ and $10^{13} \mathrm{~s}^{-1}$, respectively, from the corresponding Arrhenius plot (Figure 2). The decomposition of PPCs occurs through the cleavage of the labile peroxygen bond. First order decompositon kinetics reveal that there is no induced decomposition as observed ${ }^{5}$ for other type of PPCs. Furthermore, the similar values ${ }^{5}$ of $k_{\mathrm{d}}, E_{\mathrm{a}}$, and $A$ to other PCs based on aliphatic isocyanates indicates that substitution on the cyclohexyl ring did not have significant effect 
H. YILDIRIM et al.

Table I. Bulk polymerization of methylstyrene by polymeric peroxycarbamete

\begin{tabular}{cccccccc}
\hline Run No. & $\begin{array}{c}\text { Time } \\
(\mathrm{min})\end{array}$ & $\begin{array}{c}{[\mathrm{I}]_{0} \times 10^{4}} \\
\left(\mathrm{~mol} \mathrm{l}^{-1}\right)\end{array}$ & $\begin{array}{c}{[\mathrm{M}]_{0}} \\
\left(\mathrm{~mol} \mathrm{l}^{-1}\right)\end{array}$ & $\begin{array}{c}\text { Yield } \\
\left(\mathrm{wt}^{\circ}\right)\end{array}$ & $\begin{array}{c}R_{\mathrm{p}} \times 10^{4} \\
\left(\mathrm{~mol} \mathrm{1}^{-1} \mathrm{~s}^{-1}\right)\end{array}$ & $1 / \bar{P}_{n} \times 10^{4}$ & $\bar{M}_{n}$ \\
\hline 1 & 250 & 2.62 & 7.57 & 7.18 & 0.35 & 1.78 & 664130 \\
2 & 240 & 2.95 & 7.59 & 8.55 & 0.43 & 2.06 & 573060 \\
3 & 150 & 8.18 & 7.53 & 6.67 & 0.54 & 2.53 & 467670 \\
4 & 180 & 13.20 & 7.53 & 9.48 & 0.64 & 3.01 & 392820 \\
5 & 100 & 26.84 & 7.44 & 7.35 & 0.89 & 3.62 & 326170 \\
6 & 70 & 37.10 & 7.46 & 5.52 & 0.96 & 3.92 & 301600 \\
7 & 60 & 59.25 & 7.50 & 6.05 & 1.23 & 4.05 & 292070 \\
8 & 50 & 62.96 & 7.51 & 5.29 & 1.28 & 4.17 & 283240 \\
9 & 71 & 73.76 & 7.52 & 8.11 & 1.39 & 4.58 & 258230 \\
10 & 80 & 85.01 & 7.52 & 9.73 & 1.48 & 5.62 & 210260 \\
\hline
\end{tabular}

$[\mathrm{I}]_{0}=$ initiator concentration; $[\mathrm{M}]_{0}=$ monomer concentration.

on these parameters.

PPC was utilized as a free radical initiator for the bulk polymerization of methylstyrene at $80^{\circ} \mathrm{C}$. Typical results are shown in Table $\mathrm{I}$. Figure 3 illustrates the GPC chromatograms for polymers obtained by using different concentrations of PPC. It is interesting to note that although the molecular weights of the polymers decreased, the polydispersity of the polymers did not change by increasing the PPC concentration in the polymerization indicating the absence of chain transfer to the initiator. Polydispersity values greater than 1.5 may be due to some termination by disproportionation and chain transfer to small molecules such as monomer as suggested by Mutschler et $a l^{12}$ for $p$-methylstyrene polymerization initiated by AIBN. The radical initiation and polymerization rate is given ${ }^{13.14}$ by:

$$
R_{i}=2 f k_{\mathrm{d}}(\mathrm{I})
$$

and

$$
R_{\mathrm{p}}=k_{\mathrm{p}}(\mathrm{M})\left(f k_{\mathrm{d}}(\mathrm{I}) / k_{\mathrm{t}}\right)^{1 / 2}
$$

where (I) and (M) are the initiator and monomer concentrations and $k_{\mathrm{d}}, k_{\mathrm{p}}$, and $k_{\mathrm{t}}$ are the thermal decomposition, polymerization and termination rate constants, respectively, and $f$ is the initiator efficiency. The term $k_{\mathrm{p}}{ }^{2}$ $\left(f k_{\mathrm{d}} / k_{\mathrm{t}}\right)$ is generally denoted as $K^{2}$, which is a measure of the initiator reactivity. The value of

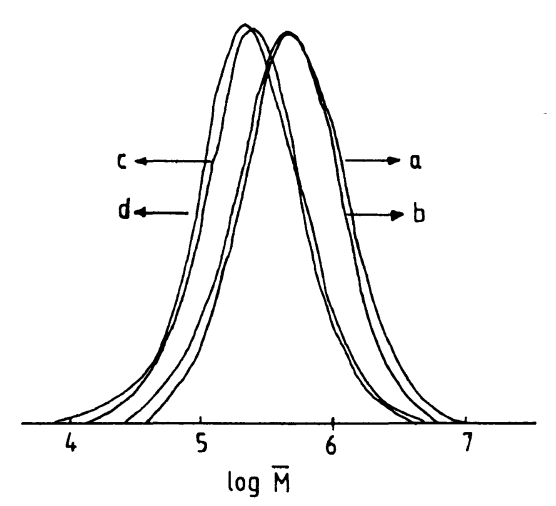

Figure 3. GPC chromatograms of poly(methylstyrene) obtained by using different polymeric peroxycarbamate concentrations. PPC $\left(\mathrm{mol} \mathrm{l}^{-1}\right)(\mathrm{a}), 2.6 \times 10^{-4}$ $\left(\bar{M}_{w} / \bar{M}_{n}=2.42\right) ; \quad(\mathrm{b}), 8.1 \times 10^{-4} \quad\left(\bar{M}_{w} / \bar{M}_{n}=2.32\right) ; \quad$ (c), $5.9 \times 10^{-3} \quad\left(\bar{M}_{w} / \bar{M}_{n}=2.34\right) ; \quad(\mathrm{d}), \quad 7.3 \times 10^{-3} \quad\left(\bar{M}_{w} / \bar{M}_{n}=\right.$ 2.35).

$K^{2}$ can be obtained from the slope of the plot of $R_{\mathrm{p}}{ }^{2} \operatorname{versus}(\mathrm{M})^{2}(\mathrm{I})$ according to eq 3 . In this study, the value of $K^{2}$ was estimated to be $4.17 \times 10^{-8} 1 \mathrm{~mol}^{-1} \mathrm{~s}^{-2}$ from Figure 4 .

For free radical, bulk polymerization the inverse degree of polymerization $\left(1 / \bar{P}_{n}\right)$ is given ${ }^{13}$ by:

$$
\frac{1}{\bar{P}_{n}}=\frac{k_{\mathrm{t}}}{k_{\mathrm{p}}{ }^{2}(\mathrm{M})^{2}} \cdot R_{\mathrm{p}}+C_{\mathrm{M}}+\frac{C_{\mathrm{l}}}{K^{2}(\mathrm{M})^{3}}
$$

where $C_{\mathrm{M}}$ and $C_{\mathrm{I}}$ represent the chain transferto-monomer and chain transfer-to-initiator constants, respectively. If $C_{\mathrm{I}}=0$, a plot of $1 / \bar{P}_{n}$ 


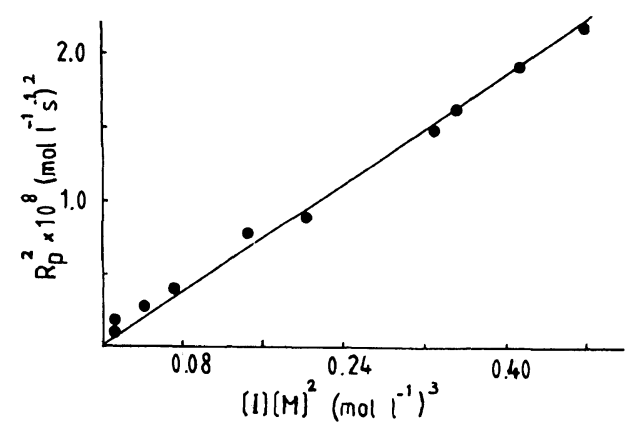

Figure 4. Plot of $R_{\mathrm{p}}$ versus $(\mathrm{M})^{2}(\mathrm{I})$ according to eq 3 for methylstyrene polymerization in bulk at $80^{\circ} \mathrm{C}$, initiated by polymeric peroxycarbamate.

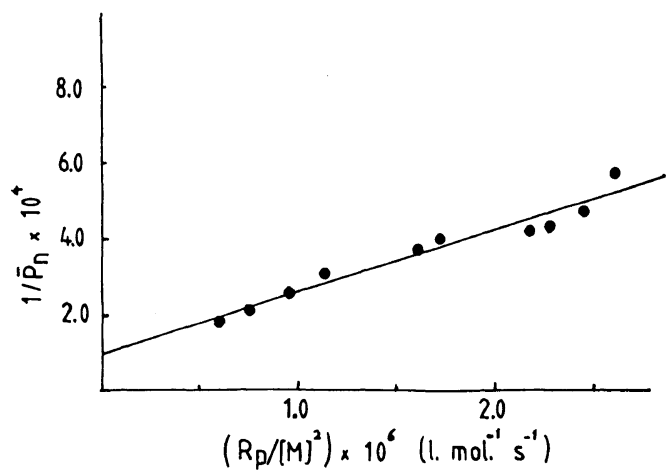

Figure 5. Plot of $1 / \bar{P}_{n}$ versus $R_{\mathrm{p}} /(\mathrm{M})^{2}$ according to eq 4 for methylstyrene polymerization in bulk at $80^{\circ} \mathrm{C}$, initiated by polymeric peroxycarbamate.

against $R_{\mathrm{p}} /(\mathrm{M})^{2}$ should yield a straight line. Such a plot is shown in Figure 5 for PPC initiated methylstyrene polymerization. The straight line obtained, up to the conversions studied, indicates that there is no chain transfer to the initiator. The intercept of this straight line gives $C_{\mathrm{M}}$. For our system, it was determined to be $8.5 \times 10^{-5}$ and was in very good agreement with literature values given for polystyrene samples. The values of $K^{2}$ and $R_{i} /(\mathrm{I})$ for the AIBN initiated polymerization of $p$-methylstyrene are $2.5 \times 10^{-8} 1 \mathrm{~mol}^{-1} \mathrm{~s}^{-2}$ and $4.46 \times 10^{-3} \mathrm{~s}^{-1}$, respectively, as obtained from the data of Muncshler et al. ${ }^{12}$ It should be noted that PPC contains more than one labile group. Therefore, dividing the $R_{i} /(\mathrm{I})$ value obtained for PPC by 6 , which is the peroxygen number in PPC, gives a value of $6.27 \times 10^{-6}$ per decomposing peroxygen bond. The comparision of this value to AIBN indicates that PPC shows slower initiation at $80^{\circ} \mathrm{C}$, therefore PPC can be used as an effective high temperature initiator.

Acknowledgment. The auothors would like to thank Dr. L. Shlegel (Hahn-Meitner-Institut, Berlin, FRG,) for the GPC measurements.

\section{REFERENCES}

1. B. M. Baysal, W. T. Short, and A. V. Tobolsky, J. Polym. Sci., A-1, 10, 909 (1972).

2. B. M. Baysal, E. H. Orhan, and I. Yilgor, J. Polym. Sci., Polym. Symp., 46, 237 (1974).

3. E. H. Orhan, I. Yilgor, and B. M. Baysal, Polymer, 18, 286 (1977).

4. Y. Yagci, M. Acar, G. Hizal, H. Yildirim, and B. M. Baysal, Angew. Makromol. Chem., 154, 169 (1987).

5. I. Yilgor and B. M, Baysal, Makromol. Chem., 186, 463 (1985).

6. B. Hazer and B. M. Baysal, Polymer, 27, 961 (1986).

7. H. Yildirim and B. M. Baysal, manuscript in preparation.

8. D. D. Perrin, W. L. F. Armerago, and D. R. Perrin, "Purification of Laboratory Chemicals," Pergamon Press, London, 1966.

9. D. J. David and H. B. Stanley, "Analytical Chemistry of Polyurethanes," High Polymer Series, XVI, Part III, Interscience, New York, N.Y., 1969, p 88 .

10. A. V. Tobolsky and R. B. Mesrobian, "Organic Peroxides," Interscience, New York, N.Y., 1954, p 53.

11. J. Brandrup and E. H. Immergut, "Polymer Handbook," Interscience Publishers, 2nd ed, 1967, p IV-17.

12. H. Mutschler, U. Schroder, E. Fahner, K. H. Ebert, and A. E. Hamielec Polymer, 26, 935 (1985).

13. F. R. Mayo, R. A. Gregg, and M. S. Matheson, $J$. Am. Chem. Soc., 73, 1651 (1951).

14. B. M. Baysal and A. V. Tobolsky, J. Phym. Sci., 8, 529 (1952). 